\title{
Development and validation of an automated algorithm to evaluate the abundance of bubbles in small bowel capsule endoscopy
}

\section{(ㄷ)(ㅇ) $\Theta$}

\author{
Authors \\ Institutions \\ 1 APHP Saint Antoine Hospital, Department of \\ Hepatogastroenterology, Paris, France \\ 2 ETIS, ENSEA, Cergy-Pontoise University, Cergy-Pontoise, \\ France \\ 3 Sorbonne University, Paris, France \\ 4 College of Arts and Sciences, Drexel University, \\ Philadelphia, Pennsylvania, United States
}

Olivia Pietri ${ }^{1}$, Gada Rezgui ${ }^{2}$, Aymeric Histace ${ }^{2}$, Marine Camus ${ }^{1,3}$, Isabelle Nion-Larmurier ${ }^{1}$, Cynthia Li ${ }^{1,4}$, Aymeric Becq ${ }^{1}$, Einas Abou Ali ${ }^{1}$, Olivier Romain ${ }^{2}$, Ulriikka Chaput ${ }^{1}$, Philippe Marteau ${ }^{1,2}$, Christian Florent ${ }^{1,2}$, Xavier Dray $^{1,2,3}$

submitted 10.8.2017

accepted after revision 3.1 .2018

\author{
Bibliography \\ DOI https://doi.org/10.1055/a-0573-1044 | \\ Endoscopy International Open 2018; 06: E462-E469 \\ (c) Georg Thieme Verlag KG Stuttgart · New York \\ ISSN 2364-3722
}

Corresponding author

Xavier Dray, Paris 6 University \& APHP, Hôpital Saint

Antoine - Digestive Diseases, 184 rue du Faubourg Saint

Antoine, Paris 75012, France

Fax: (+33)149282970

xavier.dray@aphp.fr

\section{ABSTRACT}

Background and study aims Bubbles can impair visualization of the small bowel (SB) mucosa during capsule endoscopy (CE). We aimed to develop and validate a com- puted algorithm that would allow evaluation of the abundance of bubbles in SB-CE still frames.

Patients and methods Two sets of 200 SB-CE normal still frames were created. Two experienced SB-CE readers analyzed both sets of images twice, in a random order. Each still frame was categorized as presenting with $<10 \%$ or $\geq 10 \%$ of bubbles. Reproducibility ( $\mathrm{k}$ ), sensitivity (Se), specificity (Sp), receiver operating characteristic curve, and calculation time were measured for different algorithms (Grey-level of co-occurrence matrix [GLCM], fractal dimension, Hough transform, and speeded-up robust features [SURF]) using the experts' analysis as reference. Algorithms with highest reproducibility, Se and Sp were then selected for a validation step on the second set of frames. Criteria for validation were $\mathrm{k}=1$, Se $\geq 90 \%$, Sp $\geq 85 \%$, and a calculation time $<1$ second.

Results Both SURF and GLCM algorithms had high operating points (Se and Sp over $90 \%$ ) and a perfect reproducibility $(\kappa=1)$. The validation step showed the GLCM detector strategy had the best diagnostic performances, with a Se of $95.79 \%$, a Sp of $95.19 \%$, and a calculation time of 0.037 seconds per frame.

Conclusion A computed algorithm based on a GLCM detector strategy had high diagnostic performance allowing assessment of the abundance of bubbles in SB-CE still frames. This algorithm could be of interest for clinical use (quality reporting) and for research purposes (objective comparison tool of different preparations).

\section{Introduction}

Capsule endoscopy (CE) has had a major influence in small bowel (SB) exploration since its inception in 2000 [1]. The SB is seen entirely in over $80 \%$ of CE procedures. The diagnostic yield of SB-CE is approximately $60 \%$ in the setting of obscure gastrointestinal bleeding (OGIB), and $50 \%$ in suspected Crohn's disease [2].

Residues, debris, bile, chyme, and bubbles may decrease the diagnostic yield by impairing mucosal visualization. Prior SB preparation by oral purge seems to improve the visibility of the mucosa as well as the completion rate $[3,4]$. However, its effect on bubbles residing in the SB lumen is unknown.

In the setting of colonoscopy, the Boston Bowel Preparation Scale allows an adequate report on whether the colonic mucosa is appropriately visualized during the procedure [5]. However, there is no validated cleansing score in SB-CE, which would allow determination of whether the procedure is reliable. Consequently, a reliable evaluation of SB mucosal cleansing is diffi- 
cult, and the level of evidence for efficacy of any SB-CE preparation remains low.

Attempts have been made to develop a computed SB cleansing score. Many of these scores are based on a colorimetric index, such as the red on green ratio [6,7]. For instance, Van Weyenberg et al. [6] published the first study using a computed quantitative scale to assess mucosal visibility during SB-CE, based on a colorimetric index of the tissue color bar. Although the scale was promising, presence of bubbles was not taken into account in that study. It has been suggested that anti-bubble agents, such as simethicone, given in a liquid form $(80 \mathrm{mg}$ to $600 \mathrm{mg}$ ), 20 minutes before capsule ingestion, may enhance mucosal visualization in SB-CE. However, these agents do not significantly increase the procedure's diagnostic yield [4, 813]. Moreover, validated methods to assess the amount of bubbles in CE recordings are lacking, which implies that data on anti-bubble agents are from low-quality evidence. Studies on SB cleaning scores suggest that grading scales should focus on quantitative rather than qualitative criteria to achieve better reproducibility. Therefore, we believe that an objective, reproducible score assessing the amount of bubbles in SB-CE is needed for both clinical practice and research purposes [14].

The aim of this study was to develop and validate a computed method, which would allow assessment of the abundance of bubbles covering the surface of still images.

\section{Patients and methods}

\section{Inclusion criteria}

Eligible participants presented with OGIB, indicating the need for a SB-CE procedure. SB-CE videos were edited so that only the part between the first image of the duodenum and the last one of the ileum was kept for analysis. The videos were then converted into mpeg files and included in the study.

\section{Exclusion criteria}

Patients were excluded if the SB-CE was a first- or a secondgeneration capsule, if the procedure was incomplete, or if lesions of any kind were observed.

\section{SB-CE procedure}

Bowel preparation consisted of a clear liquid diet on the day prior to the procedure, followed by split ingestion of $1.5 \mathrm{~L}$ of polyethylene glycol-electrolyte (PEG) lavage solution: $1 \mathrm{~L}$ the evening before and $0.5 \mathrm{~L}$ on the morning of the procedure day. Metoclopramide $(10 \mathrm{mg})$ could be administered orally if the capsule was delayed in the stomach (>1 hour) [15]. The procedure was complete when the capsule was expelled into the cecum. The third-generation capsule system used in this study consisted of the ingestible SB3 (PillCam, Medtronic, Minnesota, United States).

\section{Image databases}

The first dataset was created using 200 SB-CE separate, nonconsecutive, and non-clustered still frames exported from 45 SB-CE video sequences. The dataset was divided into five groups consisting of 40 still frames each. Each group was cre- ated based on the surface of mucosa (in percentage) that could not properly be examined because it was covered with bubbles. This first analysis was performed by OP. Group A comprised still frames with less than $2 \%$ bubbles, group B comprised still frames with $2 \%$ to $10 \%$ bubbles, group C comprised still frames with $10 \%$ to $25 \%$ bubbles, group D comprised still frames with $25 \%$ to $50 \%$ bubbles, and group E comprised still frames with $50 \%$ to $100 \%$ bubbles. Fig. 1 and $>$ Fig. 2 show the variety of bubble abundance on different still frames. The 200 still frames were shuffled and analyzed by two experienced readers (CF, $X D)$, blinded to the results of all the computed analysis performed on these still frames. The expert readers had previously analyzed over 500 SB-CE procedures. This review was performed twice for all 200 still frames, in random order. Each still frame was categorized based on the estimated percentage of bubbles covering the image (whether these bubbles were large or small and numerous or few), with a $10 \%$ threshold. [16, 17]. Still frames presenting with fewer than $10 \%$ bubbles were classified as "scarce in bubbles". Conversely, still frames presenting with $10 \%$ or more bubbles were classified as "abundant in bubbles". In cases of discrepancy after initial analysis (still frame classified twice as "scarce in bubbles," and twice as "abundant in bubbles"), an agreement was reached between the two readers and the still frame categorized. A second dataset was created using 200 different separate, non-consecutive, and nonclustered SB-CE still frames, divided into five groups and subsequently analyzed using the same process.

\section{Computed algorithms}

Four different computed algorithms, recognizing circular patterns, were tested in terms of their ability to adequately categorize each still frame based on the amount of bubbles, lower or higher than $10 \%$ (i. e. in the "scarce in bubbles" or "abundant in bubbles" groups) using a similar binary classification. These tests were performed using the MATLAB software (MathWorks, Natick, Massachusetts, United States). The four algorithms were divided into two groups, depending on the type of results generated. The first group included two feature-based methods, since the output of each method ("scarce in bubbles" or "abundant in bubbles") depended on a computed threshold, based on a particular characteristic of the image. The second group included two methods allowing quantification of the actual surface of the still frame presenting with bubbles. Each still-frame was categorized ("scarce in bubbles" or "abundant in bubbles") using the same bubble abundance threshold of $10 \%$.

\section{Group 1: Feature-based methods}

The first algorithm (algorithm 1) was based on the contrast computed from the Grey Level Co-occurrence matrix (GLCM) of the still frame. Classic, easy-to-compute, textural features with good discrimination capacities were obtained using parameters extracted from the GLCM of a greyscale image. Element i,j of the $256 \times 256$ matrix of an 8 -bit image (presenting with 256 grey levels for luminance) presents a high value when neighboring pixels of the same i,j value consistently appear in the considered image. Usually, this is the horizontal-right pixel 

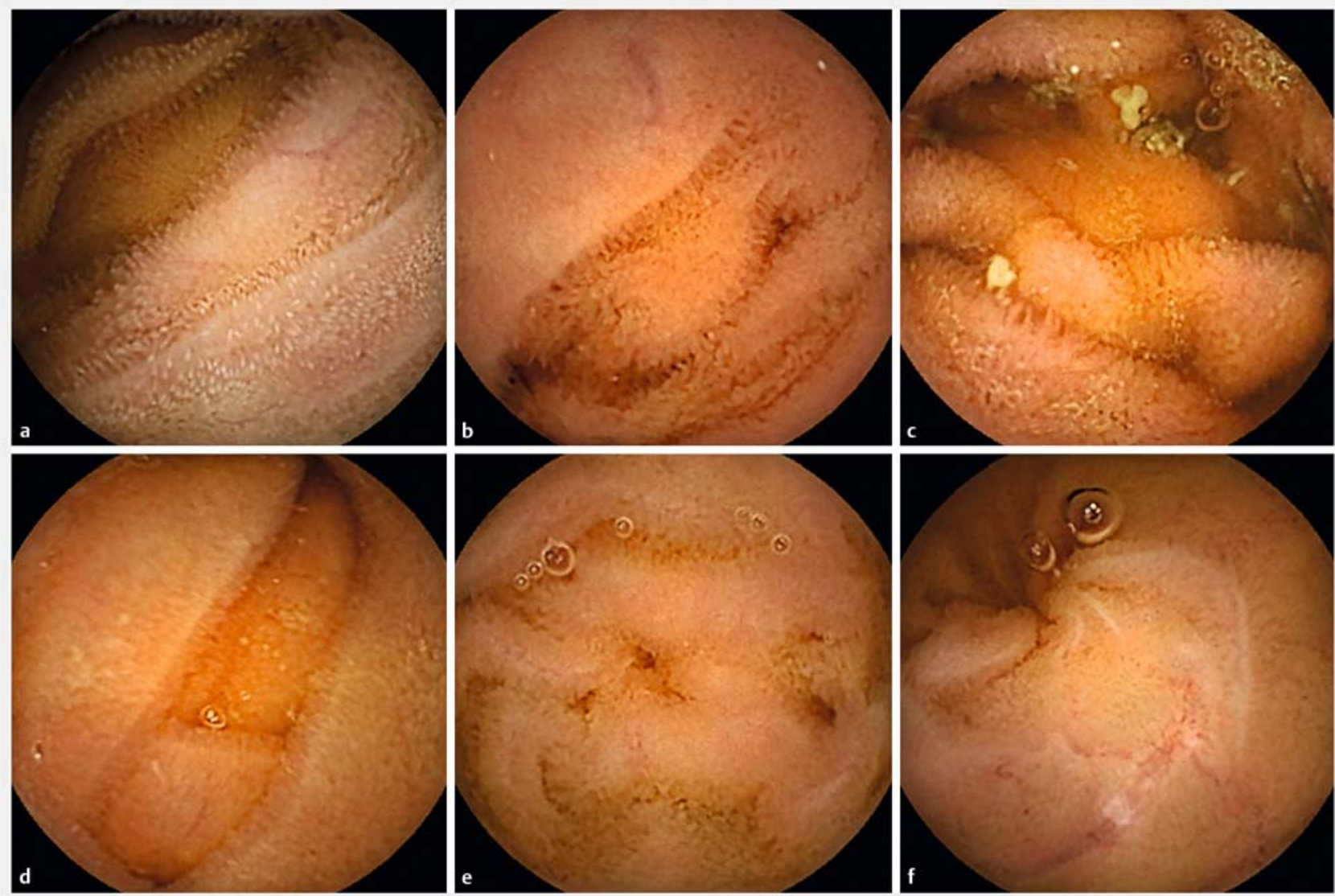

Fig. 1 Set of still frames categorized in the "scarce in bubbles" group, according to expert analysis. Images a, b and c represent still frames with less than $2 \%$ of bubbles. Images $\mathbf{d}$, e and $\mathbf{f}$ represent still frames with 2 to $10 \%$ of bubbles.

with a distance of 1 pixel, which is considered to compute the matrix. From this matrix, we computed the contrast parameter of good discrimination capacity when considering repetitive texture-like bubbles. Overall, algorithm 1 was a thresholdbased algorithm: first the contrast from GLCM of each still frame was computed, then a threshold of contrast value was applied to decide whether bubbles were present in a significant manner or not.

The second algorithm (algorithm 2) was based on similar principles, but the fractal dimension (FD) was used as a texture parameter instead of the GLCM contrast. The FD of an image strongly depends on replication of a unique pattern, at different scales, in an infinite way, to form a signal or an image-like small replicative bubbles texture. Algorithm 2 is also threshold-based: still frames were categorized based on presence of bubbles in a significant manner or not, depending on the FD of these still frames.

\section{Group 2: Surface-based methods}

The principle of surface-based methods is to quantify the actual surface of the still frame covered with bubbles and to compare the obtained results with the expert readers' binary classification.
The third algorithm (algorithm 3) was based on a Hough transform (HT) [18], which recognizes various geometrical forms, including circles, in digital images. HT increases the probability of identifying pixels within an image (contour), which would be the center $C$ of a circle with a radius $R$, using gradient information, computed with a classic edge filter strategy, such as Canny filter. In our study, intervals for $\mathrm{R}$ values were chosen in accordance with the usual size of a bubble, constrained to a maximum value of one-third of the still frame width. Once circles are detected, they are used to compute the corresponding surface within the still frame.

The fourth algorithm (algorithm 4) was based on a speededup robust features (SURF) detector strategy [19]. The principle is to extract saliency points (also known as SURF points) from the grey-level image, assuming that the geometry of a bubble and the texture of the circular surrounding environment (for a given radius $R$ ) leads to a significant increase in number of SURF points when compared to a "normal" area. When the amount of surrounding SURF points is above a given, manually tuned threshold, a saliency point is considered to belong to a bubble.

Each still frame was categorized as "scarce in bubbles" or "abundant in bubbles" after analysis by all the algorithms, based on the abundance of bubbles (fewer or more than $10 \%$ 

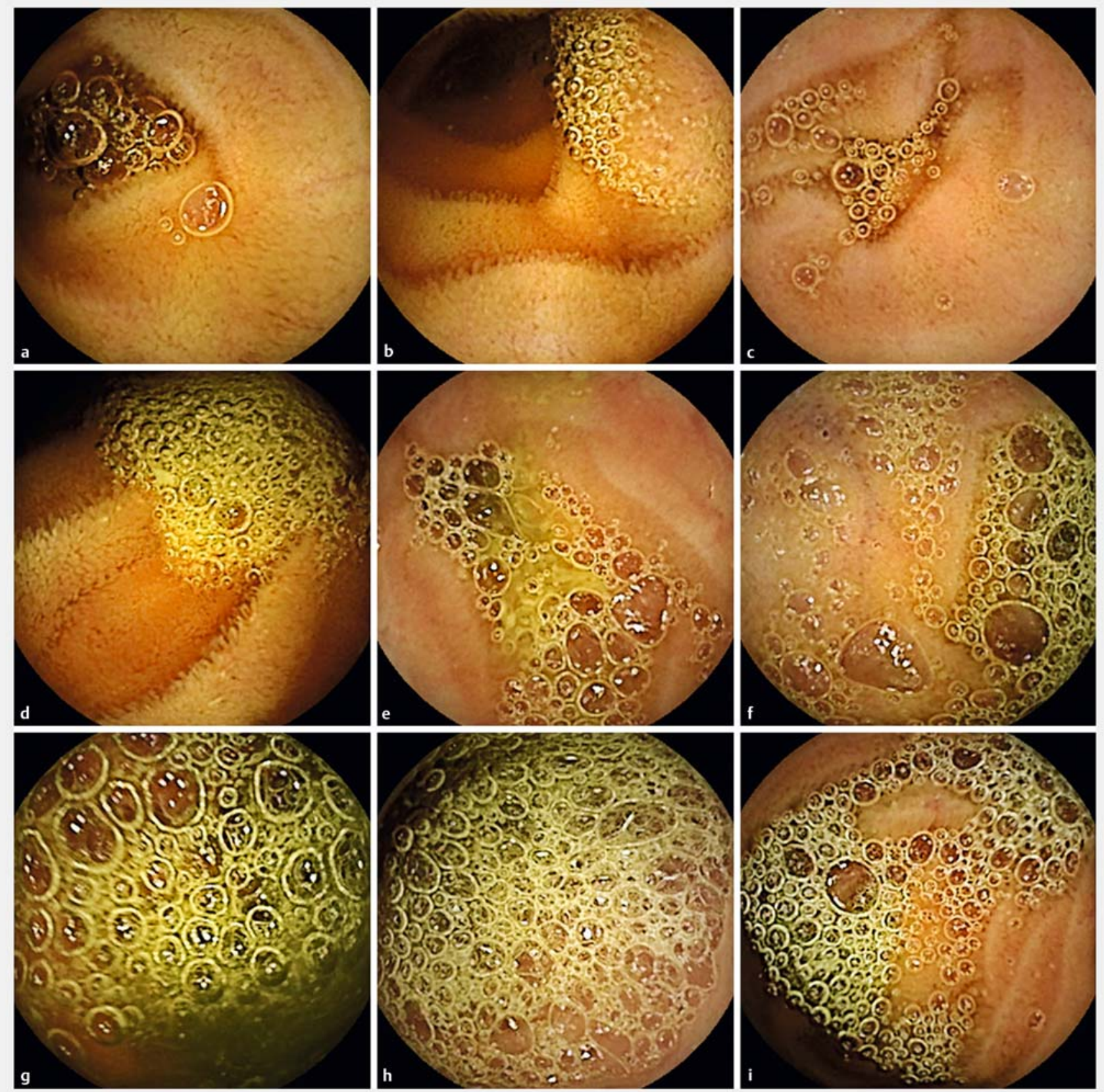

- Fig. 2 Set of still frames categorized in the "abundant in bubbles" group, according to expert analysis. Images a, b and $\mathbf{c}$ represent still frames with 10 to $25 \%$ of bubbles. Images D, E and F represent still frames with 25 to $50 \%$ of bubbles. Images $\mathbf{g}$, $\mathbf{h}$ and $\mathbf{i}$ represent still frames with 50 to $100 \%$ of bubbles.

of bubbles), comparable to the threshold used during the expert analysis.

\section{Statistical analysis}

Development step

$\mathrm{K}$ statistics were used to assess interobserver and intraobserver agreements regarding the expert analysis, as well as reproducibility of the algorithmic results. According to standards, $\mathrm{k}$ values between 0.00 and 0.20 indicate poor agreement, $\mathrm{k}$ values between 0.21 and 0.40 indicate fair agreement, $\mathrm{k}$ values between 0.41 and 0.60 indicate moderate agreement, $\mathrm{k}$ values between 0.61 and 0.80 indicate good agreement, $\mathrm{k}$ values between 0.81 and 0.99 indicate excellent agreement, and $\mathrm{a} \mathrm{k}$ value of 1.00 indicates perfect agreement [20].

The first dataset (200 images) was analyzed, using the binary classification ("scarce in bubbles" or "abundant in bubbles" groups) produced by expert readers as reference. Calculation time, receiver operating characteristic (ROC) curve, area under ROC curve (AUROCC), as well as sensitivity (Se), specificity (Sp), 
negative predictive value (NPV), and positive predictive value (PPV) of the optimal operating point were calculated for all four algorithms by tuning a specific parameter for each individual method (GLCM contrast value, FD value, HT sensitivity, number of SURF points).

\section{Validation step}

Algorithms with the best operating point (i.e., the highest sum of Se and Sp) were selected for further validation. Se, Sp, NPV and PPV values were calculated for the selected algorithms applied on the second dataset of 200 still frames (for cross-validation), using the binary classification ("scarce in bubbles" or "abundant in bubbles" groups) produced by two different expert readers (MC and INL) as reference. The primary endpoints for validation were $\mathrm{k}=1, \mathrm{Se} \geq 90 \%$, and $\mathrm{Sp} \geq 85 \%$. The secondary endpoint was the calculation time by frame $<1$ second.

\section{Results}

Forty-five eligible patients were included in the study, in order to create both datasets of still frames.

\section{Development step}

Concerning the first dataset, intraobserver correlation coefficients were $\mathrm{K}=0.92$ for $\mathrm{XD}$ and $\mathrm{K}=0.97$ for $C F$. The interobserver correlation coefficient was $\mathrm{K}=0.96$ for both expert analyses. The first analysis presented a discrepancy in 13 still frames (6.5\%). Eventually, 90 still frames (45\%) were categorized as "scarce in bubbles" and 110 still frames (55\%) as "abundant in bubbles". The diagnostic capabilities of all four algorithms are provided in $>$ Table 1. All algorithms achieved a 1.00 intraobserver correlation $\mathrm{k}$ coefficient and a sensitivity of $100 \%$, but specificity varied substantially. The SURF detector strategy had the highest operating point yielding the following: Se of $94.38 \%$ $(95 \% \mathrm{Cl}[91.19 \% ; 97.57 \%])$, Sp of $97.24 \%$ (95\%Cl [94.97\%; $99.51 \%])$, NPV of $95.45 \%(95 \% \mathrm{Cl}$ [92.56\%;98.34\%]), PPV of $96.55 \%(95 \% \mathrm{Cl}[94.02 \% ; 99.08 \%])$, AUROCC of 0.9897 , and a calculation time by frame of $11.47 \pm 7.11$ seconds.

- Fig. 3 shows illustrations of the obtained outputs for algorithms 3 and 4 (whereas no visual illustration can be given for threshold-based algorithms 1 and 2).

The GLCM detector strategy had similar diagnostic capabilities as compared to the SURF detection strategy: Se of $94.38 \%$ (95\%Cl [91.19\%;97.57\%]), Sp of $93.58 \%$ (95\%Cl [90.18\%; $96.98 \%])$, NPV of $95.32 \%$ (95\%Cl [92.39\%; 98.25\%]), PPV of $92.31 \%(95 \% \mathrm{Cl}[88.62 \% ; 96.00 \%])$, AUROCC of 0.9852 , and a calculation time by frame of $0.040 \pm 0.003$ seconds. Both the SURF and GLCM detector strategies were selected for validation ( Fig.4).

\section{Validation step}

In the second dataset, intraobserver correlation coefficients were $\mathrm{k}=0.96$ for both MC and INL. The interobserver correlation coefficient was $\mathrm{k}=0.89$ for the first analysis, and $\mathrm{k}=0.88$ for the second analysis. The first analysis presented a discrepancy in 68 frames (34\%). Eventually, 96 frames (48\%) were ca-

- Table 1 Sensitivity, specificity, NPV, PPV, and AUROCC of the four algorithms used to evaluate bubble abundance in small bowel capsule endoscopy still frames.

\begin{tabular}{|c|c|c|c|c|c|c|}
\hline & Sensitivity & Specificity & NPV & PPV & \multirow[t]{2}{*}{ AUROCC } & Calculation time \\
\hline & [95\%Cl] & [95\%Cl] & [95\%Cl] & {$[95 \% \mathrm{Cl}]$} & & by frame $(s$, mean $\pm S D)$ \\
\hline \multicolumn{7}{|l|}{ Development step } \\
\hline Algorithm 1: GLCM & $\begin{array}{l}94.38 \% \\
{[91.19 \% ; 97.57 \%]}\end{array}$ & $\begin{array}{l}93.58 \% \\
{[90.18 \% ; 96.98 \%]}\end{array}$ & $\begin{array}{l}95.32 \% \\
{[92.39 \% ; 98.25 \%]}\end{array}$ & $\begin{array}{l}92.31 \% \\
{[88.62 \% ; 96.00 \%]}\end{array}$ & 0.9852 & $0.040 \pm 0.003$ \\
\hline $\begin{array}{l}\text { Algorithm 2: } \\
\text { Fractal dimension }\end{array}$ & $\begin{array}{l}84.27 \% \\
{[79.22 \% ; 89.32 \%]}\end{array}$ & $\begin{array}{l}82.57 \% \\
{[77.31 \% ; 87.83 \%]}\end{array}$ & $\begin{array}{l}86.54 \% \\
{[81.81 \% ; 91.27 \%]}\end{array}$ & $\begin{array}{l}79.78 \% \\
{[74.21 \% ; 85.35 \%]}\end{array}$ & 0.9269 & $10.1 \pm 0.7$ \\
\hline $\begin{array}{l}\text { Algorithm 3: } \\
\text { Hough transform }\end{array}$ & $\begin{array}{l}85.39 \% \\
{[80.49 \% ; 90.29 \%]}\end{array}$ & $\begin{array}{l}81.65 \% \\
{[79.29 \% ; 87.01 \%]}\end{array}$ & $\begin{array}{l}87.25 \% \\
{[82.63 \% ; 91.87 \%]}\end{array}$ & $\begin{array}{l}79.17 \% \\
{[73.54 \% ; 84.80 \%]}\end{array}$ & 0.9252 & $1.45 \pm 1.2$ \\
\hline Algorithm 4: SURF & $\begin{array}{l}94.38 \% \\
{[91.19 \% ; 97.57 \%]}\end{array}$ & $\begin{array}{l}97.24 \% \\
{[94.97 \% ; 99.51 \%]}\end{array}$ & $\begin{array}{l}95.45 \% \\
{[92.56 \% ; 98.54 \%]}\end{array}$ & $\begin{array}{l}96.55 \% \\
{[94.02 \% ; 99.08 \%]}\end{array}$ & 0.9897 & $11.47 \pm 7.11$ \\
\hline \multicolumn{7}{|l|}{ Validation step } \\
\hline Algorithm 1: GLCM & $\begin{array}{l}95.79 \% \\
{[93.01 \% ; 98.57 \%]}\end{array}$ & $\begin{array}{l}95.19 \% \\
{[92.22 \% ; 98.16 \%]}\end{array}$ & $\begin{array}{l}96.12 \% \\
{[93.44 \% ; 98.80 \%]}\end{array}$ & $\begin{array}{l}94.79 \% \\
{[91.71 \% ; 97.87 \%]}\end{array}$ & & $0.037 \pm 0.005$ \\
\hline Algorithm 4: SURF & $\begin{array}{l}94.74 \% \\
{[91.65 \% ; 97.83 \%]}\end{array}$ & $\begin{array}{l}94.23 \% \\
{[91.00 \% ; 97.46 \%]}\end{array}$ & $\begin{array}{l}95.15 \% \\
{[92.17 \% ; 98.13 \%]}\end{array}$ & $\begin{array}{l}93.75 \% \\
{[90.40 \% ; 97.10 \%]}\end{array}$ & & $1.45 \pm 1.78$ \\
\hline
\end{tabular}

NPV, negative predictive value; PPV, positive predictive value; AUROCC, area under receiver operating characteristic curve; s, seconds; SD, standard deviation; GLCM, Grey-level of co-occurrence matrix; SURF, speeded-up robust features 

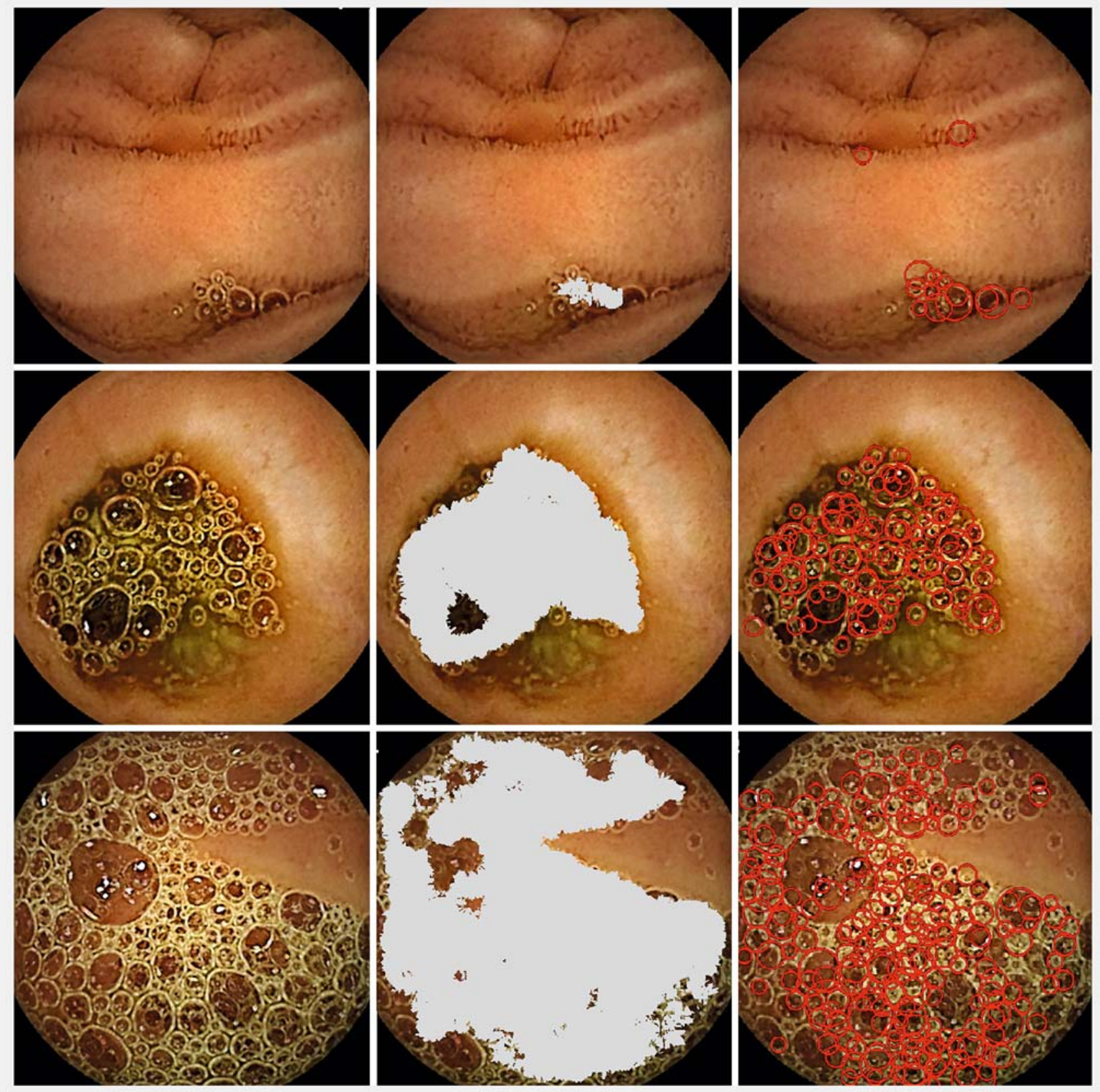

- Fig. 3 Illustrations of the outputs of surface-based methods for three different frames with various amount of bubbles: native image (left); bubbles detection based on the Grey-level of co-occurrence matrix strategy (algorithm 3, center); C bubbles detection based on the SpeededUp Robust Features SURF point detection strategy (algorithm 4, right).

tegorized as "scarce in bubbles," and 104 frames (52\%) as "abundant in bubbles". Both the SURF and the GLCM detector strategies demonstrated perfect reproducibility (intraobserver correlation $\mathrm{k}$ coefficient of 1.00). The SURF detector strategy had the following diagnostic performances: Se of $94.74 \%$ (95\% $\mathrm{Cl}[91.65 \% ; 97.83 \%])$, Sp of $94.23 \%(95 \% \mathrm{Cl}[91.00 \% ; 97.46 \%])$, NPV of $95.15 \%$ (95\% $\mathrm{Cl}[92.17 \% ; 98.13 \%])$, PPV of $93.75 \%(95 \% \mathrm{Cl}$ [90.40\%;97.10\%]). The calculation time by frame was $1.45 \pm$ 1.78 s. The GLCM detector strategy had slightly higher diagnostic capabilities: Se of $95.79 \%$ (95\%Cl [93.01\%;98.57\%]), Sp of
$95.19 \%(95 \% \mathrm{Cl}[92.22 \% ; 98.16 \%])$, NPV of $96.12 \%(95 \% \mathrm{Cl}$ [93.44\%;98.80\%]), PPV of $94.79 \%(95 \% \mathrm{Cl}[91.71 \% ; 97.87 \%])$. The calculation time by frame was significantly lower than that of the SURF detector strategy $\left(0.037 \pm 0.005\right.$ seconds $\left.\left[P<10^{-5}\right]\right)$. The GLCM detector strategy was selected as the best algorithm in terms of diagnostic performances. 


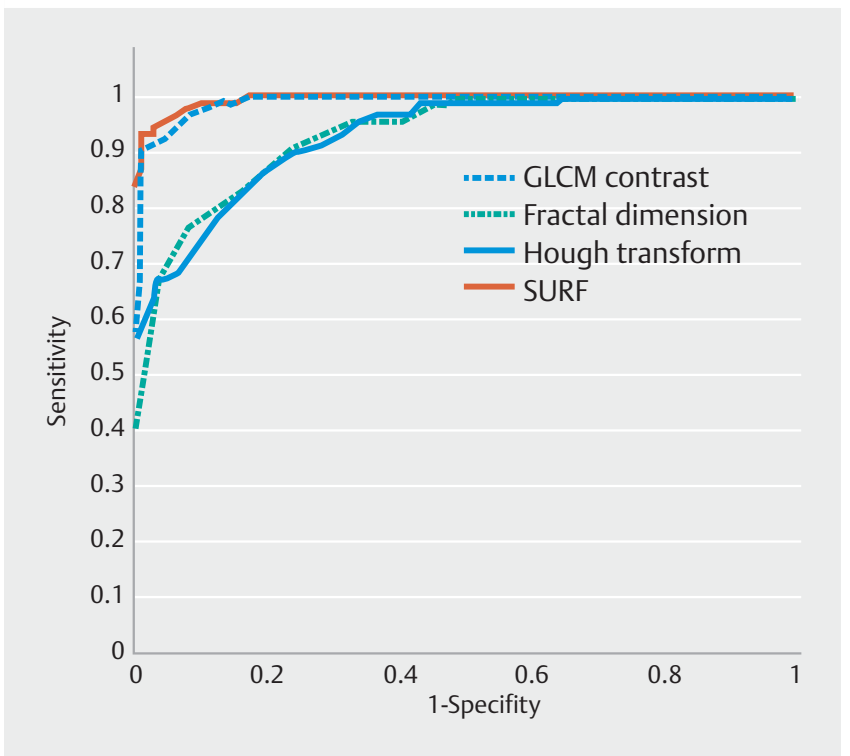

- Fig. 4 Receiver operating characteristic (ROC) curves of the four used algorithms for evaluation of bubble abundance in small bowel capsule endoscopy still frames (development step).

\section{Discussion}

We selected and validated a computed and comprehensive algorithm, allowing evaluation of the abundance of bubbles in SB-CE still frames, based on GLCM detector strategy. It proved to be perfectly reproducible $(\kappa=1.00)$, rapid $(0.037$ seconds per frame), highly sensitive (95.79\%) and specific (95.19\%).

The main strength of this study is that this algorithm allows reliable assessment of the abundance of bubbles covering the surface of SB-CE still frames. We collected 400 non-clustered images captured by the most available and most recent generation of SB-CE (SB3 PillCam system, Medtronic, Minnesota, United States). These were analyzed twice in random order by two different groups of experienced SB-CE readers blinded to the algorithmic classification. Each individual frame was categorized in a binary manner as "scarce in bubbles" or "abundant in bubbles". Cross-validation was insured by use of two independent datasets of 200 frames/se for the development and validation phases, respectively.

Establishment of a threshold of bubble abundance for which visualization of the mucosa is significantly impaired is a challenging task. A previous grading system used in three trials was deemed clinically relevant: images with no intraluminal bubble (grade 0 ) or few gas bubbles (grade 1 ) were considered as providing no limitation of interpretation $[8,9,13]$. Based on the figures from these articles and on our clinical experience, we determined that a cutoff of $10 \%$ in terms of bubble abundance on a still frame was adequate. Similarly, the only validated score used to determine the quality of visualization of the mucosa in SB-CE also considers that a visibility over $90 \%$ is optimal [16, 17]. Moreover, the chosen threshold was unchanged between the development and the validation steps of the study, for both expert analysis and algorithmic assessment. As this threshold was the same for all steps of the study, comparisons between human reading and electronic evaluation or between the four different algorithms are valid.

One may argue that a limitation of this study was that only normal SB-CE still frames were selected for analysis. However, in daily practice, most SB-CE still frames are normal, even in a video where lesions are visible on only a few still frames. Moreover, we believe that a normal examination is the most critical situation where the quality of bowel preparation should be discussed. When reporting on a normal SB-CE procedure, it is of tremendous importance to be confident in the fact that a reasonable proportion of the mucosal surface was visible throughout the whole length of the SB. In other words, SB-CE reports should clearly mention whether poor quality of preparation impaired mucosal visualization, thus hindering reliability of conclusions.

HT appeared to be a good candidate to build an algorithm, but we also assessed alternative methods. HT is computationally heavy and its processing time can be elevated when considering a large radius research interval and a large number of still frames. Moreover, circle detection sensitivity strongly correlates with the quality of the still frame and the gradient information. Thus, a low gradient level (bubble presenting a low transition intensity with the background) can lead to underestimating the surface of the bubbles. Because local textural descriptors are easy to compute and have a very low computational cost, we decided to assess a SURF one-step strategy (at a pixel level), to detect the surface occupied by bubbles. Global textural feature, such as GLCM contrast, can also provide qualitative assessment of the actual surface with bubbles within the still frame, and was expected to be a good binary estimator of bubble abundance in correlation with the physicians' visual study. The development phase showed the sensitivity of the GLMC algorithm was exactly the same as that of SURF, but the specificity of SURF was higher and the calculation time of GLCM was lower. Both algorithms, therefore, were selected for validation. GLCM eventually demonstrated higher diagnostic capabilities with significantly lower calculation time during the validation phase.

Our algorithm, based on the GLCM detector strategy, could also be useful in allowing objective evaluation of anti-bubble agents in SB-CE preparation regimens. Up to now, evaluation of the effect of anti-foaming agents on the abundance of bubbles in prospective studies has been based on expert analysis, with good - but not always excellent - agreement between experts [4, 8 - 13]. When reported, intercorrelation coefficients ( $\mathrm{K}$ or Spearman coefficients) ranged from 0.61 to 0.92 when evaluating 1 -hour videos $[8,9,13]$, and from 0.75 to 0.90 in 1 -minute videos [11]. Our study demonstrates that intercorrelation coefficients are even higher (varying between 0.88 and 0.96 ) when experts analyze individual still frames (and not entire videos). All in all, computed algorithms allow a comprehensive evaluation (each individual frame of the SB-CE procedure is analyzed) with perfect reproducibility (i.e., intraobserver correlation). Therefore, we believe that this computed algorithm, with perfect reproducibility and high sensitivity and specificity, could be used in future prospective trials (or on archived videos 
from already performed prospective trials) in an objective manner (frame-by-frame evaluation) with very high performance.

Also, the GLCM detector strategy could be combined with other criteria for evaluating SB-CE cleansing. For instance, colorimetric indexes (red/green ratio) have been used in several studies to quantify chime residues, which hinder evaluation of the mucosal surface $[6,7,20]$. We first aim to electronically define what an "appropriately cleansed still frame" is. In future studies, our goal is to develop a computed score to assess quality of SB preparation for complete SB-CE procedures. Such a tool would be of great interest in clinical practice as it would allow reliable reporting of the quality of preparation (much like the Boston Bowel Preparation Scale score in the setting of colonoscopy). Moreover, it would be helpful in clinical research, to help compare efficacy of different preparation regimens.

\section{Conclusion}

In conclusion, this study showed that a computed algorithm based on the GLCM detector strategy has a perfect reproducibility, a high diagnostic sensitivity (95.79\%) and specificity (95.19\%), and is a rapid method of analysis ( 0.037 seconds per frame), for assessment of the abundance of bubbles on SB-VCE still frames. It proved to be a powerful tool to differentiate still frames with a low amount of bubbles from those with more than $10 \%$ bubbles. This algorithm could be implemented in SB-CE reading software and used automatically for evaluation of anti-bubble agents. In the future, we aim to develop a computed SB-CE cleaning score with the help of this algorithm. Such a score would be of great interest in the fields of clinical practice and research.

\section{Competing interests}

Pr Dray has acted as a consultant for Boston Scientific, Fujifilm, Medtronic and Pentax.

\section{References}

[1] Iddan G, Meron G, Glukhovsky A et al. Wireless capsule endoscopy. Nature 2000; 405: 417

[2] Pennazio M, Spada C, Eliakim R et al. Small-bowel capsule endoscopy and device-assisted enteroscopy for diagnosis and treatment of small-bowel disorders: European Society of Gastrointestinal Endoscopy (ESGE) Clinical Guideline. Endoscopy 2015; 47: 352 - 376

[3] Yung DE, Rondonotti E, Sykes C et al. Systematic review and meta-analysis: is bowel preparation still necessary in small bowel capsule endoscopy? Expert Rev Gastroenterol Hepatol 2017; 11: 979- 993
[4] Koulaouzidis A, lakovidis DK, Karargyris A et al. Optimizing lesion detection in small-bowel capsule endoscopy: from present problems to future solutions. Expert Rev Gastroenterol Hepatol 2015; 9: 217 - 235

[5] Lai E], Calderwood AH, Doros G et al. The Boston bowel preparation scale: a valid and reliable instrument for colonoscopy-oriented research. Gastrointest Endosc 2009; 69: 620-625

[6] Van Weyenberg SJB, De Leest HTJI, Mulder CJJ. Description of a novel grading system to assess the quality of bowel preparation in video capsule endoscopy. Endoscopy 2011; 43: 406-411

[7] Abou Ali E, Histace A, Camus A et al. Development and validation of a highly sensitive and highly specific computed assessment of cleansing score for small bowel capsule endoscopy. United European Gastroenterol ] 2016; 4: A604

[8] Albert ], Göbel CM, Lesske J et al. Simethicone for small bowel preparation for capsule endoscopy: a systematic, single-blinded, controlled study. Gastrointest Endosc 2004; 59: 487 - 491

[9] Wu L, Cao Y, Liao C et al. Systematic review and meta-analysis of randomized controlled trials of Simethicone for gastrointestinal endoscopic visibility. Scand J Gastroenterol 2011; 46: 227 - 235

[10] Fang YH, Chen CX, Zhang BL. Effect of small bowel preparation with simethicone on capsule endoscopy. J Zhejiang Univ Sci B 2009; 10: $46-51$

[11] Spada C, Riccioni ME, Familiari P et al. Polyethylene glycol plus simethicone in small-bowel preparation for capsule endoscopy. Dig Liver Dis 2010; 42: $365-370$

[12] Rosa BJ, Barbosa M, Magalhaes ] et al. Oral purgative and simethicone before small bowel capsule endoscopy. World J Gastrointest Endosc 2013; 5: $67-73$

[13] Papamichael K, Karatzas P, Theodoropoulos I et al. Simethicone adjunct to polyethylene glycol improves small bowel capsule endoscopy imaging in non-Crohn's disease patients. Ann Gastroenterol 2015; 28: $464-468$

[14] Keuchel M, Kurniawan N, Baltes P et al. Quantitative measurements in capsule endoscopy. Comput Biol Med 2015; 65: 333-347

[15] Koulaouzidis A, Giannakou A, Yung DE et al. Do prokinetics influence the completion rate in small-bowel capsule endoscopy? A systematic review and meta-analysis Curr Med Res Opin 2013 Sep29: 1171 1185

[16] Brotz C, Nandi N, Conn M et al. A validation study of 3 grading systems to evaluate small-bowel cleansing for wireless capsule endoscopy: a quantitative index, a qualitative evaluation, and an overall adequacy assessment. Gastrointest Endosc 2009; 69: 262 - 270

[17] Klein A, Gizbar M, Bourke MJ et al. Validated computed cleansing score for videocapsule endoscopy. Dig Endosc 2016; 28: 564 - 569

[18] Hough PVC. Method and Means for Recognizing Complex Patterns. US Patent 3,069,654 1962: Ser. No. 17,7156 Claims

[19] Vilarino F, Malagelada C, Azpiroz F et al. Categorization and segmentation of intestinal content frames for wireless capsule endoscopy. IEEE Trans Inf Technol Biomed 2012; 16: 1341 - 1352

[20] Landis JR, Koch GG. The measurement of observer agreement for categorical data. Biometrics 1977; 33: 159-174 